\title{
Habit or Utility: A Key Choice Point in Promoting the Adoption of Telehealth in China
}

\author{
Yun Fan $\mathbb{D}^{1,2}$ Sifeng Liu, ${ }^{1}$ Jun Liu, ${ }^{2}$ Saad Ahmed Javed $\mathbb{D},{ }^{3}$ and Zhigeng Fang $\mathbb{D}^{1}$ \\ ${ }^{1}$ College of Economics and Management, Nanjing University of Aeronautics and Astronautics, Nanjing 211106, China \\ ${ }^{2}$ Nantong University, Nantong 226019, China \\ ${ }^{3}$ Institute for Grey Systems and Decision Sciences, GreySys Foundation, Lahore, Pakistan \\ Correspondence should be addressed to Zhigeng Fang; zhigengfang@163.com
}

Received 25 March 2020; Revised 15 May 2020; Accepted 2 June 2020; Published 4 July 2020

Guest Editor: Lei Xie

Copyright (c) 2020 Yun Fan et al. This is an open access article distributed under the Creative Commons Attribution License, which permits unrestricted use, distribution, and reproduction in any medium, provided the original work is properly cited.

\begin{abstract}
Telehealth, as an indispensable means of technical support in the Healthy China Strategy, currently has less than 20 percent adoption rate in China despite a great deal of government policies and investments. In the current study, to analyse the influencing factors behind doctors' and patients' adoption of telehealth, an asymmetric dynamic evolutionary game model of doctor-patient behaviour selection was established. Based on the model solution, the evolutionarily stable strategies that emerge in different situations were analysed. The results show that it is difficult for the adoption of telehealth in China to keep pace with coverage due to the "dual low" nature of telehealth: both doctors' utility from telehealth and patients' telehealth cost threshold are too low to incentivize adoption. The strategy to promote the adoption of telehealth in China should include providing adequate training for doctors and patients on the use of telehealth technology, rewarding doctors who provide telehealth services and raising the threshold cost of patient's telehealth adoption.
\end{abstract}

\section{Introduction}

Telehealth is the provision of medical services remotely through the internet via mobile phones, smartphones, computers, tablets, or other wireless mobile devices $[1,2]$. Telehealth can overcome the limitations of geographical distance and allow the provision of professional technical guidance in situations characterized by a shortage of doctors. It can be used to educate local doctors with limited case exposure and reduce patients' medical expenses by reducing their travel burden. At the same time, internet technology can help link patient data and examination results, thereby avoiding repeated examinations of patients and saving medical resources [3-6].

According to a World Health Organization (WHO) survey in 2015, with the development of information and communication technologies (ICT) and the popularization of mobile Internet technology, approximately $3 / 4 \quad(n=51$; $73 \%$ ) of countries that participated in the survey reported including telehealth as a policy or strategic goal $[7,8]$. The United States government sees telehealth as an important measure to promote low-cost, high-quality medical system reform [2]. The British and Japanese governments have also adopted telehealth (virtual visits) as part of their health care reforms [9]. The Chinese government has included telehealth in its Healthy China Strategy and expects to achieve win-win outcomes for hospitals and patients in both urban and rural areas through telehealth $[10,11]$. Although countries are eagerly developing telehealth, the actual adoption rate of this service is uneven. In 2016, 61 percent of medical institutions and 40 to 50 percent of hospitals in the United States used some form of telehealth [7]. A survey across nine Latin American nations during the same period found that the use of telehealth ranged from 25 percent of hospitals in Colombia to 65 percent of hospitals in Chile [12]. In China, the utilization rate of telehealth was still lower than 20 percent in 2019 [13].

With shortages of doctors and unbalanced medical and health resources in urban and rural areas, the health field in 
China is facing new challenges, such as an ageing population and changes in the disease spectrum [14-16]. As an essential strategy to break the deadlock, the Chinese government has fully deployed telehealth while completing the "leap-forward" development of the country's communications infrastructure $[17,18]$. At present, investment in telehealth in China ranks second in the world, and telehealth has been initiated in all first-class hospitals, covering all poor counties $[9,19]$. By the end of 2020, all primary medical and health institutions in the medical consortium should be covered [20]. However, despite its inherent advantages, the adoption of telehealth is still not high, although the Chinese government invests a great deal in telehealth policies and funds, with the most obvious challenge being the inability or reluctance of doctors and patients to adopt telehealth.

The literature indicates that telehealth is a complex system [12]. Whether telehealth can be used effectively as an information technology depends on the acceptance and use of this technology by users $[21,22]$. The factors influencing the acceptability of telehealth to doctors and patients, primary users, and stakeholders of telehealth, have been widely studied in developed countries and some developing countries by using various theoretical models [23]. However, in countries with a strong traditional medical culture, such as China, the main barrier is the conflict between doctors' and patients' established habits and telehealth objectives $[5,24]$. The literature that considers the behaviour and evolution of doctor and patient adoption of telehealth from a habit and utility perspective is negligible. The underlying reason doctors and patients have not adopted telehealth is that their individual estimates of telehealth utility are lower than their estimates of the utility of habitual in-person visits. For individuals, due to constraints such as imperfect information, the choice of not adopting telehealth is superficially rational but in fact irrational from the perspective of collective rationality $[25,26]$. Therefore, there is a dire need to study the key factors that affect the adoption of telehealth among doctors and patients, specifically with reference to modes of telehealth and in-person visits, the utility of these visit modes, and how doctors and patients choose and evolve between these modes. To this end, evolutionary game theory provides a suitable approach, combining game theory and dynamic evolution to study the stable structure of a game system and the behavioural strategy selection process of a subject in the process of evolution by introducing a dynamic mechanism [27-29]. In this study, we constructed an evolutionary model of doctor-patient behaviour choice to evaluate how to improve the utility of telehealth for doctors and patients, making greater use of the telehealth mode relative to the in-person mode so that doctors and patients can break habits and become telehealth users.

\section{Theoretical Background}

The existing literature has explored the factors that influence the adoption of telehealth by doctors and patients from different perspectives. Many scholars have approached this question from the perspective of information technology acceptance. Dünnebeil et al. and Liu et al. used the technology acceptance model (TAM) to study the acceptance of medical information systems by doctors or patients in terms of both perceived ease of use and perceived usefulness $[30,31]$. Some scholars have extended the TAM model. Tsai integrated the TAM model, social capital theory, and social cognition theory to develop a comprehensive behavioural model for analysing the telehealth usage intention of elderly people [32]. Rho et al. developed the Telemedicine Service Acceptance (TSA) model based on the TAM model, emphasizing the importance of incentives, and verified the effectiveness of TSA in explaining doctors' acceptance of telehealth services [33]. Based on the TAM model and combined with contract theory, Wang studied the influencing factors of patient telehealth service quality, price, waiting time, transportation cost, and so on [34]. Zhou et al. used an extended TAM model to verify that medical affordability, waiting time, and information quality are decisive variables affecting acceptance of telehealth among elderly people [9].

In general, the TAM model focuses on extrinsic motivation, such as the user's perceived ease of use, and involves less consideration of intrinsic motivation [34]. For this reason, Venkatesh et al. integrated eight user acceptance models, including TAM, the motivational model (MM) and the theory of reasoned action (TRA), etc., and constructed their unified theory of acceptance and use of technology (UTAUT) with strong explanatory power [35]. According to the UTAUT, individuals' behavioural intentions in using technology are jointly determined by performance expectancy, effort expectancy, social influence, and facilitating conditions [36]. Diño et al. and Adenuga et al. used the UTAUT model to determine the effectiveness of behavioural intention for telehealth use among elderly people and doctors and revealed the importance of doctors' motivation $[21,23]$.

From the perspective of game theory, Rajan et al. combined a nonatomic game with a queuing model and studied the speed-quality trade-off of medical operations by analysing the doctor's utility (price, equilibrium arrival rate, etc.) and the patient's utility (reward from seeking treatment, congestion cost, payment, etc.) [37]. After constructing an evolutionary model of a game between hospitals and patients and analysing factors such as medical expenses, reimbursement ratio of medical insurance, and hospital costs, Wang et al. and Zhan et al. highlighted the importance of improving the utility of patients' telehealth services and the reimbursement ratio of medical insurance [38, 39].

In light of this literature review and empirical observations, this study underscores that resistance to telehealth is common. Doctors are accustomed to face-to-face visits, and their refusal to use telehealth does not affect their income. Instead, adoption of telehealth consumes their energy and resources, as they need to spend time and energy familiarizing themselves with the new system $[5,22,40]$. Therefore, many scholars have emphasized the importance of motivating doctors to use telehealth [7, 23, 33]. Ordinary patients, influenced by traditional Chinese medicine's concepts of "looking, smelling, questioning, and cutting," are used to in-person visits, as intangible telehealth appears 
less effective than an in-person visit; moreover, the habitual in-person model also has lower fees $[5,22,40]$.

According to the literature, the factors influencing doctors' and patients' adoption of telehealth are summarized in Table 1.

As seen from Table 1, the reward from visits, travel burden, payment, waiting cost, perceived ease of use, and perceived usefulness are the main factors influencing patients' adoption of telehealth. Remuneration, incentives, perceived ease of use, and perceived usefulness are the main factors influencing doctors' adoption of telehealth. Some of these factors, such as perceived ease of use and perceived usefulness, relate to the user's intention to use telehealth systems rather than the utility from actual adoption [32]. To convert intentionality into the utility of adopting telehealth, the cost of patient's telehealth learning and the fixed cost of related equipment are introduced to reflect perceived ease of use and perceived usefulness, because patients are less likely to learn to use telehealth if they are unwilling to avail themselves of telehealth services. Once patients begin to receive telehealth services, they will invest their efforts in learning to verify the ease of use and usefulness of telehealth; this is patients' learning cost of telehealth. When patients find telehealth easy to use and useful, they will take action to pay for the network connection, cameras, or other facilities required for the use of telehealth services, as well as the cost of operation, and thus bear certain amortized costs. Similarly, since Chinese doctors must provide telehealth services due to an administrative order, the usefulness of telehealth has spread among doctors, whereas the government or the hospital bears the cost of related equipment, and thus doctors' learning cost of telehealth is used to reflect the perceived ease of use of the service [19]. Therefore, in this article, the reward from visits, travel burden, payment, telehealth learning cost, amortized costs, and waiting cost are taken as the main influencing factors behind patients' adoption of telehealth, and variables including remuneration, telehealth learning cost, and incentives are taken as the main influencing factors behind doctors' adoption of telehealth.

\section{Methods}

3.1. Analysis Framework. The basic idea of evolutionary game theory is that in a group of a certain size, both sides of the game are driven by bounded rationality, and it is impossible to find the optimal equilibrium point in every game. Instead, through repeated games, that is, continually imitating the dominant strategies of themselves and others in the past, all players will tend toward a particular stable strategy through long-term improvement [27-29].

In this study, whether doctors and patients adopt telehealth in practice can be regarded as the result of a game between doctors and patients, and the two sides of the game do not benefit symmetrically from the adoption, so it is an asymmetric evolutionary game. In addition, due to this information asymmetry and the bounded rationality of both sides of the game, it is difficult for doctors and patients to know whether their decisions are in line with the requirements of profit maximization when they make their decisions. Instead, they continually improve by imitating their own and others' dominant strategies in the past. Furthermore, strategy improvements are not adopted by all players at the same time but by gradual adjustment based on differences in the bounded rationality levels of the players. The rate of strategy adjustment can be expressed by the evolutionary dynamic equation of biological evolution - the gene replication dynamic formula [41]. Therefore, the analysis framework of the current study is an asymmetric replication dynamic evolutionary game, that is, a game in which a member is randomly selected from the two groups of doctors and patients repeatedly to assess the behavioural selection.

3.2. Strategy Combinations. Through long-term observation of the behavioural choices of doctors and patients during visits, we summarized four strategy combinations: (1) doctors and patients did not use telehealth but used in-person visits; (2) patients used telehealth, but doctors did not; thus although inperson visits were eventually used, patients paid the learning cost of using telehealth; (3) doctors used telehealth, but patients did not, and although in-person visits were finally used, doctors paid the learning cost of using telehealth; (4) doctors and patients all adopted telehealth, but not all diagnoses and treatments, such as laboratory or in-person clinical examinations, could be performed through telehealth. Therefore, in practice, a combination of both telehealth and in-person visits was mostly adopted. At this point, doctors paid the learning cost of using telehealth, and patients paid both the learning cost and the amortized cost of using telehealth.

3.3. Payoff Matrix of the Doctor-Patient Game. Let $i$ represent in-person visits and $t$ telehealth visits.

In strategy one, when the government set the price per visit based on the level of hospitals and doctors, the utility of each doctor was related to the number of patients treated per unit of time, that is, the doctor's service rate and the commission on each visit fee. The doctor's utility function can be expressed as

$$
R_{\mathrm{i}}=\gamma_{\mathrm{i}} p_{\mathrm{i}} \mu
$$

Each patient's utility comprised a reward from the visit, a travel burden, a payment, and a waiting cost. The most significant advantage of telehealth is that patients do not need to travel. To simulate the travel burden of face-to-face visits, we modelled the travel burden $\delta B_{(\mathrm{d})}$ as a function of the distance $d$ between patients and doctors. $\delta$ is a random variable related to traffic congestion. The lower the amount of traffic, the smaller is the value of $\delta, 0<\delta<1$. The patient's actual payment is related to the medical insurance rate and the prices charged by the government. The patient's utility function can be expressed as

$$
\psi_{\mathrm{i}}=m_{\mathrm{i}}-\delta B_{(\mathrm{d})}-\beta_{\mathrm{i}} p_{\mathrm{i}}-E_{\mathrm{i}} .
$$

In strategy two, patients paid the learning cost of using telehealth, and in strategy three, doctors paid the learning 
TABLE 1: Factors influencing the adoption of telehealth by doctors and patients.

\begin{tabular}{|c|c|c|c|}
\hline Author(s) (year) & Influencing factors & Object of study & Model/theory \\
\hline $\begin{array}{l}\text { Dunnebeil et al. (2012) [30], } \\
\text { Liu et al. (2013) [31] }\end{array}$ & Perceived ease of use, perceived usefulness & $\begin{array}{c}\text { Doctors/ } \\
\text { patients } \\
\end{array}$ & TAM \\
\hline Tsai (2014) [32] & $\begin{array}{l}\text { Perceived ease of use, perceived usefulness social capital theory, } \\
\text { social cognition theory }\end{array}$ & Elderly people & \multirow{4}{*}{ Extended TAM } \\
\hline Rho et al. (2014) [33] & $\begin{array}{l}\text { Perceived incentives, clinical factors, individual factors, } \\
\text { perceived ease of use, perceived usefulness }\end{array}$ & Doctors & \\
\hline Wang (2016) [34] & \multirow{2}{*}{$\begin{array}{l}\text { Service quality, price, waiting time, transportation cost, etc. } \\
\text { Satisfaction with medical services (MSS) (affordability, waiting } \\
\text { time), perceived ease of use, information quality }\end{array}$} & Patients & \\
\hline Zhou et al. (2019) [9] & & Elderly people & \\
\hline Diño and de Guzman (2015) [21] & \multirow{2}{*}{$\begin{array}{l}\text { Performance expectancy, effort expectancy, social influence } \\
\text { Suitable incentives, performance expectancy, effort expectancy, } \\
\text { facilitating condition }\end{array}$} & Elderly people & \multirow[b]{2}{*}{ UTAUT } \\
\hline Adenuga et al. (2017) [23] & & Doctors & \\
\hline $\begin{array}{l}\text { Wang et al. (2015) [38], Zhan } \\
\text { et al. (2017) [39] }\end{array}$ & $\begin{array}{l}\text { Utility of patients' telehealth services, medical expenses, } \\
\text { reimbursement ratio of medical insurance, hospital costs, etc. }\end{array}$ & $\begin{array}{l}\text { Hospitals and } \\
\text { patients }\end{array}$ & \multirow[b]{2}{*}{ Game theory } \\
\hline Rajan et al. (2019) [37] & $\begin{array}{l}\text { Doctor's utility (price, equilibrium arrival rate, etc.), patient's } \\
\text { utility (reward from seeking treatment, congestion cost, } \\
\text { payment, etc.) }\end{array}$ & $\begin{array}{l}\text { Doctors and } \\
\text { patients }\end{array}$ & \\
\hline $\begin{array}{l}\text { Xue and Liang (2007) [22], } \\
\text { Combi et al. (2016) [5] }\end{array}$ & $\begin{array}{l}\text { Doctor: face-to-face visit habits, extra cost of telehealth, etc. } \\
\text { Patient: cost of telehealth, reimbursement, etc. }\end{array}$ & $\begin{array}{l}\text { Doctors and } \\
\text { patients }\end{array}$ & \multirow{3}{*}{$\begin{array}{l}\text { Literature review, } \\
\text { survey, report, etc. }\end{array}$} \\
\hline $\begin{array}{l}\text { U.S. Department of Health and } \\
\text { Human Services }(2016)[7,22]\end{array}$ & Payment, especially more comprehensive coverage by Medicare & Policies & \\
\hline Scott Kruse et al. (2018) [40] & $\begin{array}{c}\text { Technically challenged staff, resistance to change, cost, } \\
\text { reimbursement, etc. }\end{array}$ & $\begin{array}{l}\text { Doctors and } \\
\text { patients }\end{array}$ & \\
\hline
\end{tabular}

cost of using telehealth. Strategy four, as mentioned earlier, involved the combination of telehealth and in-person visits and was often used in practice. Assuming that the clinical feasibility of telehealth is $\alpha, \alpha \in(0,1)$, the doctor's utility is the sum of the utility of telehealth and in-person visits. At this time, the doctor's utility function can be expressed as

$$
R_{\mathrm{t}}=\alpha \gamma_{\mathrm{t}} p_{\mathrm{t}} \mu+(1-\alpha) \gamma_{\mathrm{i}} p_{\mathrm{i}} \mu-S_{\mathrm{q}}
$$

Similarly, the patient's utility is the sum of the utility in the two modes. As an advantage of telehealth, patients do not have to travel, so their travel burden is zero. At the same time, patients need to bear the learning cost and amortized cost of using telehealth. The patient's utility function can be expressed as

$$
\begin{aligned}
\psi_{\mathrm{t}}= & \alpha m_{\mathrm{t}}+(1-\alpha)\left(m_{\mathrm{i}}-\delta B_{(\mathrm{d})}\right)-S_{\mathrm{v}}-C \\
& -\alpha \beta_{\mathrm{t}} p_{\mathrm{t}}-(1-\alpha) \beta_{\mathrm{i}} p_{\mathrm{i}}-\alpha E_{\mathrm{t}}-(1-\alpha) E_{\mathrm{i}}
\end{aligned}
$$

To study whether to reward doctors for using telehealth, we assumed that doctors would be rewarded for their use of telehealth regardless of whether patients agreed or disagreed to adopt the service. According to equations (1)-(4), we constructed the benefits of the four strategy combinations. The payoff matrix of the two players is shown in Table 2, and the main parameters and their meanings are shown in Table 3.

\subsection{Replication Dynamic Equation of the Doctor-Patient} Game. It is assumed that the proportion of doctors in the "adoption" game party was $x(0 \leq x \leq 1)$, and there were $1-$ $x$ doctors in the "no adoption" game party. Similarly, the proportion of patients in the "adoption" game party was $y(0 \leq y \leq 1)$, and there were $1-y$ patients in the "no adoption" game party. According to evolutionary game theory, when the profit of a strategy is higher than the average profit of a mixed strategy, this strategy will develop in the group and be more likely to be adopted. The replication dynamic equation is a dynamic differential equation that describes the frequency at which a particular strategy is adopted in a population [28]. Let $U_{11}$ represent the benefits of doctors choosing to adopt the telehealth strategy, $U_{12}$ the benefits of doctors choosing not to adopt the telehealth strategy, and $\bar{U}_{1}$ the average benefits of doctors:

$$
\begin{aligned}
U_{11}= & y\left[\alpha \gamma_{\mathrm{t}} p_{\mathrm{t}} \mu+(1-\alpha) \gamma_{\mathrm{i}} p_{\mathrm{i}} \mu-S_{\mathrm{q}}+w\right] \\
& +(1-y)\left(\gamma_{\mathrm{i}} p_{\mathrm{i}} \mu-S_{\mathrm{q}}+w\right) \\
= & y \alpha \gamma_{\mathrm{t}} p_{\mathrm{t}} \mu-y \alpha \gamma_{\mathrm{i}} p_{\mathrm{i}} \mu+\gamma_{\mathrm{i}} p_{\mathrm{i}} \mu-S_{\mathrm{q}}+w \\
U_{12}= & y\left(\gamma_{\mathrm{i}} p_{\mathrm{i}} \mu\right)+(1-y)\left(\gamma_{\mathrm{i}} p_{\mathrm{i}} \mu\right) \\
= & \gamma_{\mathrm{i}} p_{\mathrm{i}} \mu \\
\bar{U}_{1}= & x U_{11}+(1-x) U_{12} \\
= & x\left(y \alpha \gamma_{\mathrm{t}} p_{\mathrm{t}} \mu-y \alpha \gamma_{\mathrm{i}} p_{\mathrm{i}} \mu+\gamma_{\mathrm{i}} p_{\mathrm{i}} \mu-S_{\mathrm{q}}+w\right) \\
& +(1-x)\left(\gamma_{\mathrm{i}} p_{\mathrm{i}} \mu\right) \\
= & x y \alpha \gamma_{\mathrm{t}} p_{\mathrm{t}} \mu-x y \alpha \gamma_{\mathrm{i}} p_{\mathrm{i}} \mu-x S_{\mathrm{q}}+x w+\gamma_{\mathrm{i}} p_{\mathrm{i}} \mu .
\end{aligned}
$$

Let $U_{21}$ represent the benefits of patients choosing to adopt the telehealth strategy, $U_{22}$ the benefits of patients choosing not to adopt the telehealth strategy, and $\bar{U}_{2}$ the average benefits of patients: 
TABle 2: Payoff matrix of the doctor-patient game.

\begin{tabular}{lccc}
\hline & & Game party II : patient & No adoption \\
& & Adoption & $\gamma_{\mathrm{i}} p_{\mathrm{i}} \mu-S_{\mathrm{q}}+w$ \\
Game party I: doctor & Adoption & $\alpha m_{\mathrm{t}}+(1-\alpha)\left(m_{\mathrm{i}}-\delta B_{(\mathrm{d})}\right)-S_{\mathrm{v}}-C-\alpha \beta_{\mathrm{t}} p_{\mathrm{t}}-(1-\alpha) \beta_{\mathrm{i}} p_{\mathrm{i}}-\alpha E_{\mathrm{t}}-(1-\alpha) E_{\mathrm{i}}$ & $m_{\mathrm{i}}-\delta B_{(\mathrm{d})}-\beta_{\mathrm{i}} p_{\mathrm{i}}-E_{\mathrm{i}}$ \\
& & $\gamma_{\mathrm{i}} p_{\mathrm{i}} \mu$ & $\gamma_{\mathrm{i}} p_{\mathrm{i}} \mu$ \\
& & $m_{\mathrm{i}}-\delta B_{(\mathrm{d})}-S_{\mathrm{v}}-\beta_{\mathrm{i}} p_{\mathrm{i}}-E_{\mathrm{i}}$ & $m_{\mathrm{i}}-\delta B_{(\mathrm{d})}-\beta_{\mathrm{i}} p_{\mathrm{i}}-E_{\mathrm{i}}$ \\
& & &
\end{tabular}

TABLE 3: Main parameters and their meanings.

\begin{tabular}{|c|c|}
\hline Parameter & Meaning \\
\hline$R$ & Doctor's utility \\
\hline$\psi$ & Patient's utility \\
\hline$d$ & Distance of the patient from the doctor \\
\hline$\delta B_{(\mathrm{d})}$ & Patient's travel burden from distance $d$ to the doctor ( $\delta$ is a random variable, $0<\delta<1$ ) \\
\hline$m$ & Patient's reward for each visit \\
\hline$m_{\mathrm{i}}$ & Patient's reward for each in-person visit \\
\hline$m_{\mathrm{t}}$ & Patient's reward for each telehealth visit \\
\hline$\mu$ & Doctor's service rate, that is, the number of patients treated per unit of time \\
\hline$p$ & Price per visit charged by the government \\
\hline$p_{\mathrm{i}}$ & Price per visit charged by the government for an in-person visit \\
\hline$p_{\mathrm{t}}$ & Price per visit charged by the government for telehealth \\
\hline$\beta$ & Medical insurance rate, $0 \leq \beta \leq 1$ \\
\hline$\beta_{\mathrm{i}}$ & Medical insurance rate for an in-person visit \\
\hline$\beta_{\mathrm{t}}$ & Medical insurance rate for telehealth \\
\hline$\gamma$ & Doctor's commission rate on each visit fee \\
\hline$\gamma_{\mathrm{i}}$ & Doctor's commission rate on each visit fee for an in-person visit \\
\hline$\gamma_{\mathrm{t}}$ & Doctor's commission rate on each visit fee for telehealth \\
\hline$E$ & Patient's waiting costs for each visit \\
\hline$E_{\mathrm{i}}$ & Patient's waiting costs for each in-person visit \\
\hline$E_{\mathrm{t}}$ & Patient's waiting costs for each telehealth visit \\
\hline$\alpha$ & Clinical feasibility of telehealth \\
\hline C & Patient's amortized cost of telehealth adoption \\
\hline$S_{\mathrm{v}}$ & Patient's learning costs of telehealth adoption \\
\hline$S_{\mathrm{q}}$ & Doctor's learning cost of telehealth adoption \\
\hline$w^{4}$ & Doctor's rewards from telehealth adoption \\
\hline
\end{tabular}

$$
\begin{aligned}
U_{21}= & x\left[\alpha m_{\mathrm{t}}+(1-\alpha)\left(m_{\mathrm{i}}-\delta B_{(\mathrm{d})}\right)-S_{\mathrm{v}}-C-\alpha \beta_{\mathrm{t}} p_{\mathrm{t}}-(1-\alpha) \beta_{\mathrm{i}} p_{\mathrm{i}}-\alpha E_{\mathrm{t}}-(1-\alpha) E_{\mathrm{i}}\right] \\
& +(1-x)\left(m_{\mathrm{i}}-\delta B_{(\mathrm{d})}-S_{\mathrm{v}}-\beta_{\mathrm{i}} p_{\mathrm{i}}-E_{\mathrm{i}}\right) \\
= & x \alpha\left(m_{\mathrm{t}}-m_{\mathrm{i}}+\delta B_{(\mathrm{d})}-\beta_{\mathrm{t}} p_{\mathrm{t}}+\beta_{\mathrm{i}} p_{\mathrm{i}}-E_{\mathrm{t}}+E_{\mathrm{i}}\right)-x C+m_{\mathrm{i}}-\delta B_{(\mathrm{d})}-S_{\mathrm{v}}-\beta_{\mathrm{i}} p_{\mathrm{i}}-E_{\mathrm{i}}, \\
U_{22}= & x\left(m_{\mathrm{i}}-\delta B_{(\mathrm{d})}-\beta_{\mathrm{i}} p_{\mathrm{i}}-E_{\mathrm{i}}\right)+(1-x)\left(m_{\mathrm{i}}-\delta B_{(\mathrm{d})}-\beta_{\mathrm{i}} p_{\mathrm{i}}-E_{\mathrm{i}}\right) \\
= & m_{\mathrm{i}}-\delta B_{(\mathrm{d})}-\beta_{\mathrm{i}} p_{\mathrm{i}}-E_{\mathrm{i}}, \\
\bar{U}_{2}= & y U_{21}+(1-y) U_{22} \\
= & y\left[x \alpha\left(m_{\mathrm{t}}-m_{\mathrm{i}}+\delta B_{(\mathrm{d})}-\beta_{\mathrm{t}} p_{\mathrm{t}}+\beta_{\mathrm{i}} p_{\mathrm{i}}-E_{\mathrm{t}}+E_{\mathrm{i}}\right)-x C+m_{\mathrm{i}}-\delta B_{(\mathrm{d})}-S_{\mathrm{v}}-\beta_{\mathrm{i}} p_{\mathrm{i}}-E_{\mathrm{i}}\right] \\
& +(1-y)\left(m_{\mathrm{i}}-\delta B_{(\mathrm{d})}-\beta_{\mathrm{i}} p_{\mathrm{i}}-E_{\mathrm{i}}\right) \\
= & x y \alpha\left(m_{\mathrm{t}}-m_{\mathrm{i}}+\delta B_{(\mathrm{d})}-\beta_{\mathrm{t}} p_{\mathrm{t}}+\beta_{\mathrm{i}} p_{\mathrm{i}}-E_{\mathrm{t}}+E_{\mathrm{i}}\right)-x y C-y S_{\mathrm{v}}+m_{\mathrm{i}}-\delta B_{(\mathrm{d})}-\beta_{\mathrm{i}} p_{\mathrm{i}}-E_{\mathrm{i}} .
\end{aligned}
$$


The gene replication dynamic equation of the doctor groups was

$$
\begin{aligned}
F(x) & =\frac{\mathrm{d} x}{\mathrm{~d} t}=x\left(U_{11}-\bar{U}_{1}\right) \\
& =x\left[\left(y \alpha \gamma_{\mathrm{t}} p_{\mathrm{t}} \mu-y \alpha \gamma_{\mathrm{i}} p_{\mathrm{i}} \mu+\gamma_{\mathrm{i}} p_{\mathrm{i}} \mu-S_{\mathrm{q}}+w\right)-\left(x y \alpha \gamma_{\mathrm{t}} p_{\mathrm{t}} \mu-x y \alpha \gamma_{\mathrm{i}} p_{\mathrm{i}} \mu-x S_{\mathrm{q}}+x w+\gamma_{\mathrm{i}} p_{\mathrm{i}} \mu\right)\right] \\
& =x(1-x)\left(y \alpha \gamma_{\mathrm{t}} p_{\mathrm{t}} \mu-y \alpha \gamma_{\mathrm{i}} p_{\mathrm{i}} \mu-S_{\mathrm{q}}+w\right) .
\end{aligned}
$$

Similarly, the gene replication dynamic equation of patient groups was

$$
\begin{aligned}
F(y)= & \frac{\mathrm{d} y}{\mathrm{~d} t}=y\left(U_{21}-\bar{U}_{2}\right) \\
= & y\left[x \alpha\left(m_{\mathrm{t}}-m_{\mathrm{i}}+\delta B_{(\mathrm{d})}-\beta_{\mathrm{t}} p_{\mathrm{t}}+\beta_{\mathrm{i}} p_{\mathrm{i}}-E_{\mathrm{t}}+E_{\mathrm{i}}\right)-x C+m_{\mathrm{i}}-\delta B_{(\mathrm{d})}-S_{\mathrm{v}}-\beta_{\mathrm{i}} p_{\mathrm{i}}-E_{\mathrm{i}}\right. \\
& \left.-x y \alpha\left(m_{\mathrm{t}}-m_{\mathrm{i}}+\delta B_{(\mathrm{d})}-\beta_{\mathrm{t}} p_{\mathrm{t}}+\beta_{\mathrm{i}} p_{\mathrm{i}}-E_{\mathrm{t}}+E_{\mathrm{i}}\right)+x y C+y S_{\mathrm{v}}-\left(m_{\mathrm{i}}-\delta B_{(\mathrm{d})}-\beta_{\mathrm{i}} p_{\mathrm{i}}-E_{\mathrm{i}}\right)\right] \\
= & y(1-y)\left[x \alpha\left(m_{\mathrm{t}}-m_{\mathrm{i}}+\delta B_{(\mathrm{d})}-\beta_{\mathrm{t}} p_{\mathrm{t}}+\beta_{\mathrm{i}} p_{\mathrm{i}}-E_{\mathrm{t}}+E_{\mathrm{i}}\right)-x C-S_{\mathrm{v}}\right] .
\end{aligned}
$$

\section{Stability Analysis of the Evolutionary Game}

According to gene replication dynamic equations (11) and (12), let $F(x)=0$ and $F(y)=0$; five equilibrium points in the system could be obtained, named, in turn, $O(0,0), A(0$, $1), B(1,0), C(1,1)$, and $D\left(x^{*}, y^{*}\right)$. Among them, $x^{*}=$ $\left(S_{\mathrm{v}} /\left(\alpha\left(m_{\mathrm{t}}-m_{\mathrm{i}}+\delta B_{(\mathrm{d})}-\beta_{\mathrm{t}} p_{\mathrm{t}}+\beta_{\mathrm{i}} p_{\mathrm{i}}-E_{\mathrm{t}}+E_{\mathrm{i}}\right)-C\right)\right) \quad$ and
$y^{*}=\left(\left(w-S_{\mathrm{q}}\right) /\left(\alpha \mu\left(\gamma_{\mathrm{i}} p_{\mathrm{i}}-\gamma_{\mathrm{t}} p_{\mathrm{t}}\right)\right)\right)$. The equilibrium state is not necessarily stable. According to Friedman's stability theory, the stability of equilibrium points in evolutionary games can be analysed by constructing a Jacobian matrix [42]. According to equations (11) and (12), the Jacobian matrix $J$ could be obtained:

$$
\begin{aligned}
J & =\left[\begin{array}{ll}
J_{1} & J_{2} \\
J_{3} & J_{4}
\end{array}\right] \\
& =\left[\begin{array}{cc}
(1-2 x)\left(y \alpha \gamma_{\mathrm{t}} p_{\mathrm{t}} \mu-y \alpha \gamma_{\mathrm{i}} p_{\mathrm{i}} \mu-S_{\mathrm{q}}+w\right) & x(1-x)\left(\alpha \gamma_{\mathrm{t}} p_{\mathrm{t}} \mu-\alpha \gamma_{\mathrm{i}} p_{\mathrm{i}} \mu\right) \\
y(1-y)\left[\alpha\left(m_{\mathrm{t}}-m_{\mathrm{i}}+\delta B_{(\mathrm{d})}-\beta_{\mathrm{t}} p_{\mathrm{t}}+\beta_{\mathrm{i}} p_{\mathrm{i}}-E_{\mathrm{t}}+E_{\mathrm{i}}\right)-C\right] & (1-2 y)\left[x \alpha\left(m_{\mathrm{t}}-m_{\mathrm{i}}+\delta B_{(\mathrm{d})}-\beta_{\mathrm{t}} p_{\mathrm{t}}+\beta_{\mathrm{i}} p_{\mathrm{i}}-E_{\mathrm{t}}+E_{\mathrm{i}}\right)-x C-S_{\mathrm{v}}\right]
\end{array}\right] .
\end{aligned}
$$

According to equation (13), the Jacobian determinant and its trace were obtained:

$$
\begin{aligned}
\operatorname{Det} J= & J_{1} J_{4}-J_{2} J_{3} \\
= & (1-2 x)\left(y \alpha \gamma_{\mathrm{t}} p_{\mathrm{t}} \mu-y \alpha \gamma_{\mathrm{i}} p_{\mathrm{i}} \mu-S_{\mathrm{q}}+w\right)(1-2 y)\left[x \alpha\left(m_{\mathrm{t}}-m_{\mathrm{i}}+\delta B_{(\mathrm{d})}-\beta_{\mathrm{t}} p_{\mathrm{t}}+\beta_{\mathrm{i}} p_{\mathrm{i}}-E_{\mathrm{t}}+E_{\mathrm{i}}\right)-x C-S_{\mathrm{v}}\right] \\
& -x(1-x)\left(\alpha \gamma_{\mathrm{t}} p_{\mathrm{t}} \mu-\alpha \gamma_{\mathrm{i}} p_{\mathrm{i}} \mu\right) y(1-y)\left[\alpha\left(m_{\mathrm{t}}-m_{\mathrm{i}}+\delta B_{(\mathrm{d})}-\beta_{\mathrm{t}} p_{\mathrm{t}}+\beta_{\mathrm{i}} p_{\mathrm{i}}-E_{\mathrm{t}}+E_{\mathrm{i}}\right)-C\right], \\
T_{\mathrm{r}}= & J_{1}+J_{4} \\
= & (1-2 x)\left(y \alpha \gamma_{\mathrm{t}} p_{\mathrm{t}} \mu-y \alpha \gamma_{\mathrm{i}} p_{\mathrm{i}} \mu-S_{\mathrm{q}}+w\right)+(1-2 y)\left[x \alpha\left(m_{\mathrm{t}}-m_{\mathrm{i}}+\delta B_{(\mathrm{d})}-\beta_{\mathrm{t}} p_{\mathrm{t}}+\beta_{\mathrm{i}} p_{\mathrm{i}}-E_{\mathrm{t}}+E_{\mathrm{i}}\right)-x C-S_{\mathrm{v}}\right] .
\end{aligned}
$$


If and only if both conditions (16) and (17) are met at the same time, it is the equilibrium point of the replicating dynamic equation, and the the evolutionary stability strategy (ESS) is given as follows:

$$
\operatorname{det} J=J_{1} J_{4}-J_{2} J_{3}>0 \text { (Jacobian determinant condition), }
$$

$$
T_{\mathrm{r}}=J_{1}+J_{4}<0 \text { (tracecondition). }
$$

According to the above-calculated data, $T_{\mathrm{r}}=0$ at the local equilibrium point $D\left(x^{*}, y^{*}\right)$, which did not meet the trace condition. Therefore, equilibrium point $D\left(x^{*}, y^{*}\right)$ was certainly not an evolutionarily stable strategy of the system. For the remaining four equilibrium points, the local stability of the equilibrium points could be judged according to the determinant and trace values of the Jacobian matrix. The results of the local stability analysis are shown in Table 4 .

From the local stability analysis, we found that there were three ESSs at five equilibrium points in the following three situations.

Scenario one: when $w<S_{\mathrm{q}}, O(0,0)$ is an ESS. The dynamic phase diagram of the system evolution is illustrated in Figure 1. In this case, from an initial state, the system will converge to $(0,0)$; that is, neither the doctor nor the patient will adopt telehealth. For doctors, the reward for adopting telehealth is less than their learning cost. For reasons of utility, the probability of doctors adopting telehealth will gradually evolve from 1 to 0 . Moreover, since $A(0,1)$ is not a stable point, once the doctor does not use telehealth, the probability of patients using telehealth will gradually evolve from 1 to 0 .

Scenario two: when $w>S_{\mathrm{q}}$ and $\alpha\left(m_{\mathrm{t}}-m_{\mathrm{i}}+\delta B_{(\mathrm{d})}-\right.$ $\left.\beta_{\mathrm{t}} p_{\mathrm{t}}+\beta_{\mathrm{i}} p_{\mathrm{i}}-E_{\mathrm{t}}+E_{\mathrm{i}}\right)<C+S_{\mathrm{v}}, B(1,0)$ is an ESS. In this case, from an initial state, the system will converge to $(1,0)$, and the system evolution dynamic phase diagram is illustrated in Figure 2. Because for doctors, the reward of adopting telehealth is greater than their learning cost; for the consideration of utility, the probability of doctors adopting telehealth will gradually evolve from 0 to 1 . At the same time, for patients, if their learning cost and the amortized cost are greater than $\alpha\left[\delta B_{(\mathrm{d})}+\left(m_{\mathrm{i}}-m_{\mathrm{t}}\right)-\left(\beta_{\mathrm{t}} p_{\mathrm{t}}-\beta_{\mathrm{i}} p_{\mathrm{i}}\right)-\left(E_{\mathrm{t}}-E_{\mathrm{i}}\right)\right]$, for the sake of utility, the probability of patients adopting telehealth will gradually evolve from 1 to 0 .

Scenario three: when $w>S_{\mathrm{q}}+\alpha \mu\left(\gamma_{\mathrm{i}} p_{\mathrm{i}}-\gamma_{\mathrm{t}} p_{\mathrm{t}}\right)$ and $\alpha\left(m_{\mathrm{t}}-m_{\mathrm{i}}+\delta B_{(\mathrm{d})}-\beta_{\mathrm{t}} p_{\mathrm{t}}+\beta_{\mathrm{i}} p_{\mathrm{i}}-E_{\mathrm{t}}+E_{\mathrm{i}}\right)>C+S_{\mathrm{v}}, C(1,1)$ is an ESS. In this case, from an initial state, the system will converge to $(1,1)$, and its system evolution dynamic phase diagram is illustrated in Figure 3. In this case, for doctors, the reward from using telehealth is greater than $S_{\mathrm{q}}+\alpha \mu\left(\gamma_{\mathrm{i}} p_{\mathrm{i}}-\gamma_{\mathrm{t}} p_{\mathrm{t}}\right)$, and out of utility considerations, the probability of doctors adopting telehealth will gradually evolve from 0 to 1 . In scenario two, because the patients do not eventually adopt telehealth, even if the doctors are willing to use it, the visits are ultimately done in face-to-face mode, so $\alpha=0, \alpha \mu\left(\gamma_{\mathrm{i}} p_{\mathrm{i}}-\gamma_{\mathrm{t}} p_{\mathrm{t}}\right)=0$. At the same time, if patients' learning cost and amortized cost are less than $c$, for the sake of utility, their probability using telehealth will gradually evolve from 0 to 1 .

\section{Discussion}

The study proposes an asymmetric replication dynamic evolutionary game model of telehealth selection between doctors and patients. By analysing the results of the evolutionary game, we obtained evolutionarily stable strategies in different situations. When $w<S_{\mathrm{q}}$, the system is stable at $O$ $(0,0)$, meaning the utility of telehealth for doctors and patients is lower than that of in-person visits and telehealth projects are relatively difficult to promote. When $w>S_{\mathrm{q}}$ and $C+S_{\mathrm{v}}>\alpha\left(m_{\mathrm{t}}-m_{\mathrm{i}}+\delta B_{(\mathrm{d})}-\beta_{\mathrm{t}} p_{\mathrm{t}}+\beta_{\mathrm{i}} p_{\mathrm{i}}-E_{\mathrm{t}}+E_{\mathrm{i}}\right)$, the system is stable at $B(1,0)$; in this scenario, the utility of doctors from telehealth use is high, while that of patients is low. Although doctors will adopt telehealth, the utilization of telehealth cannot be improved without the participation of patients. When $w>S_{\mathrm{q}}+\alpha \mu\left(\gamma_{\mathrm{i}} p_{\mathrm{i}}-\gamma_{\mathrm{t}} p_{\mathrm{t}}\right)$ and $C+S_{\mathrm{v}}<\alpha\left(m_{\mathrm{t}}-m_{\mathrm{i}}+\delta B_{(\mathrm{d})}-\beta_{\mathrm{t}} p_{\mathrm{t}}+\beta_{\mathrm{i}} p_{\mathrm{i}}-E_{\mathrm{t}}+E_{\mathrm{i}}\right)$, the system is stable at $c(1,1)$. At this point, the utility of doctors and patients from telehealth use is higher than that from face-toface visits; thus, telehealth is adopted by both parties, and the final utilization rate is improved. Here, we discuss how to promote the adoption of telehealth by doctors and patients.

\subsection{Provide Adequate Free Training for Doctors and Patients} in Telehealth Technology. The literature indicates that the lack of training for doctors and patients is one of the barriers to telehealth adoption $[5,22,40,43,44]$. As it is based on a new information technology, telehealth adoption imposes a certain learning cost. In particular, middle-aged and elderly people affected by the digital divide face higher learning costs, regardless of whether they are doctors or patients [21]. The lack of training can increase the time and effort required for doctors and patients to learn, thus further increasing the learning cost. Research shows that telehealth and face-toface visits are complements rather than substitutes [9]. Faced with complex conditions, doctors and patients, especially doctors, will pay a considerable learning cost to master and apply their knowledge of the relevant technologies, such as when and how to use telehealth. Our study shows that when all else are equal, a high cost of learning can lead directly to doctors or patients abandoning the use of telehealth. To reduce the cost of learning for doctors and patients, adequate, free training on telehealth techniques, for example, guiding doctors and patients in deciding when to use telehealth, is needed [22]. How do doctors and patients use telehealth devices and familiarize themselves with general ICT technologies, such as PC or mobile phone operations, in the first place? Inadequate training of doctors in the use of telehealth may cause medical errors [5].

5.2. Reward Doctors. The current study shows that doctors' use of telehealth is a prerequisite for patients' use of telehealth. As Rho et al. point out, telehealth is a valuable healthcare service only when doctors adopt it proactively [33]. In China, doctors must provide telehealth due to an administrative order, which is guided by a public welfare and assistance motive, without additional remuneration [19]. In the absence of extrinsic motivators, the objective of adopting 


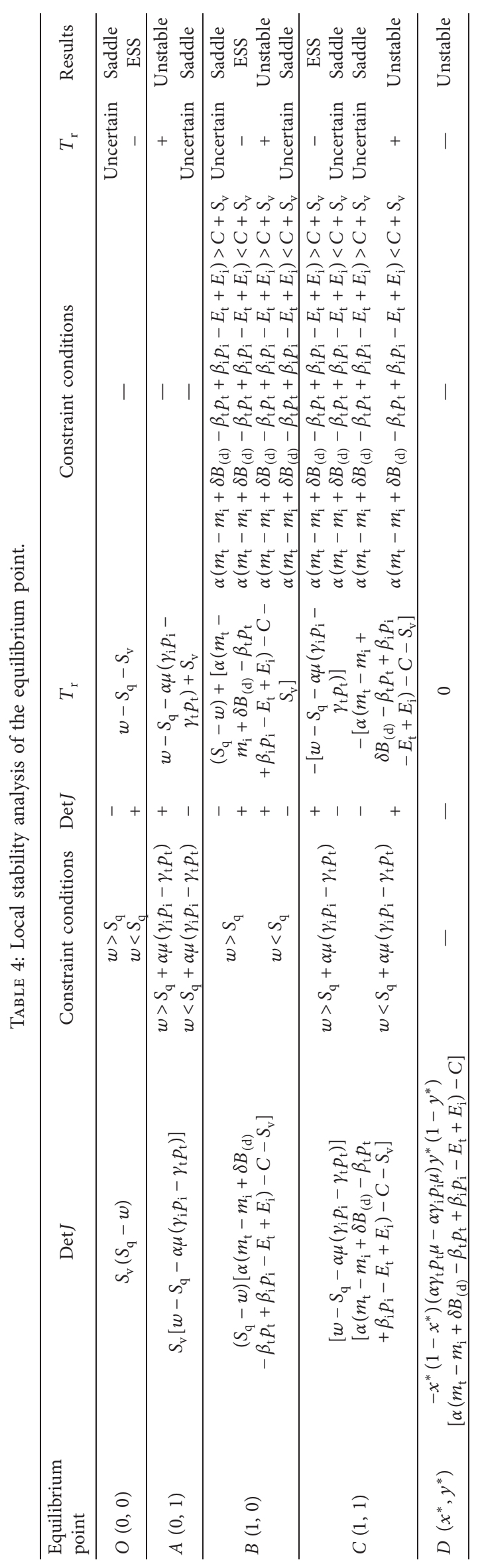




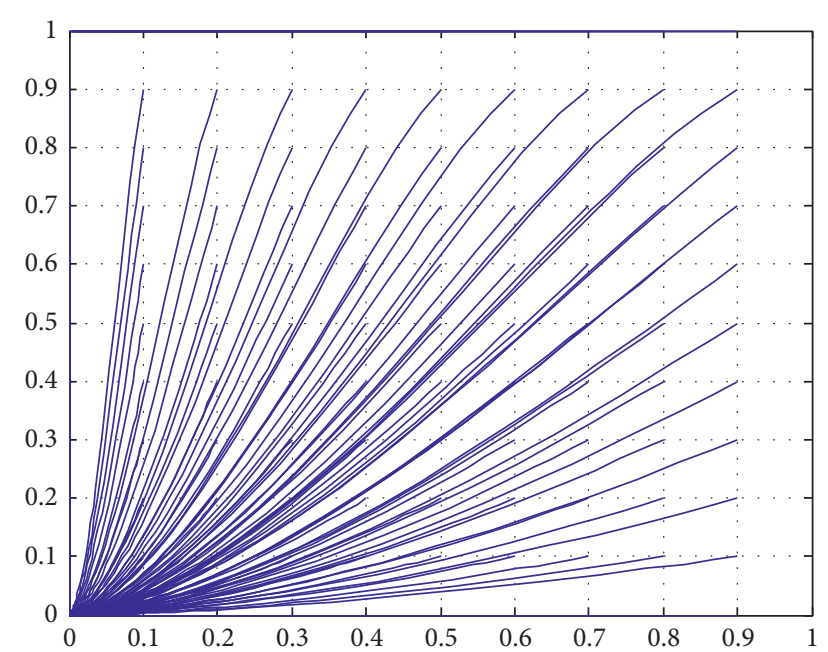

Figure 1: Dynamic phase diagram of system evolution in scenario one.

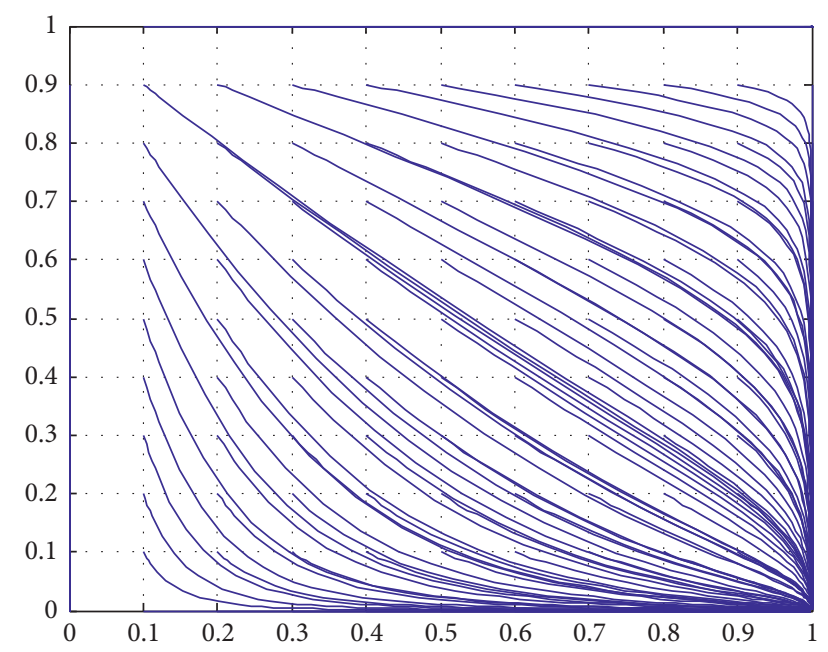

FIGURe 2: Dynamic phase diagram of system evolution in scenario two.

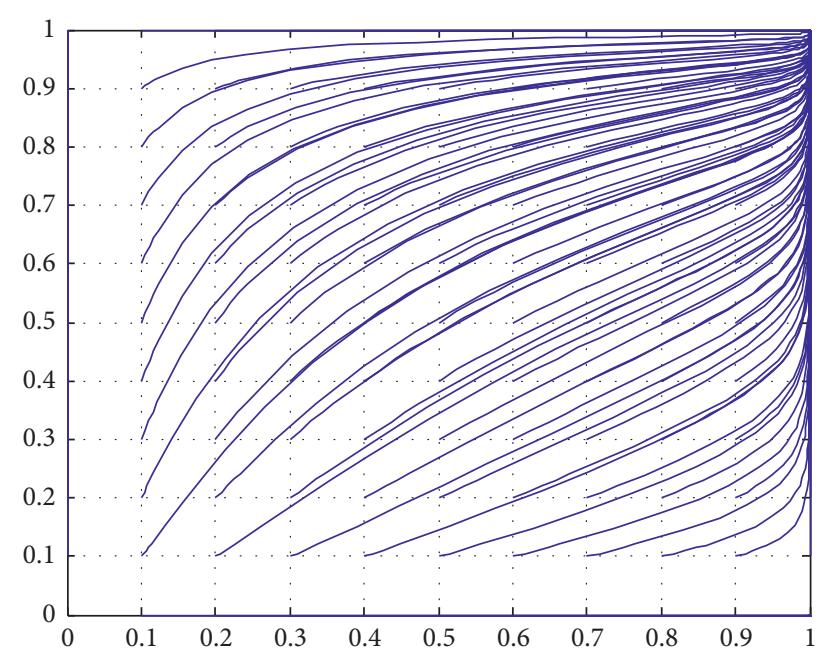

FIGURE 3: Dynamic phase diagram of system evolution in scenario three. telehealth can be achieved in the short term but may face challenges in the long term. Doctors may eventually abandon telehealth due to the lower utility they derive from it than from in-person visits. Therefore, as the literature suggests, doctors should be rewarded [5, 23, 45]. The current study is pioneering in suggesting that doctors' rewards for adopting telehealth should be greater than $S_{\mathrm{q}}+\alpha \mu\left(\gamma_{\mathrm{i}} p_{\mathrm{i}}-\gamma_{\mathrm{t}} p_{\mathrm{t}}\right)$. Further, observing the difference between doctors' face-to-face and telehealth remuneration, many scholars have suggested that doctors who adopt telehealth should be paid the same as those who conduct face-to-face visits [2, 33]. That is, the rewards for doctors should be extended until doctors who use telehealth are paid the same as those who use face-to-face visits and, consequently, no new learning costs are incurred after they receive telehealth training.

5.3. Raise the Cost Threshold for Patient Adoption of Telehealth. The cost of adopting telehealth for patients is composed of a learning cost and an amortized cost, which has a threshold value of $\alpha\left[\delta B_{(\mathrm{d})}+\left(m_{\mathrm{i}}-m_{\mathrm{t}}\right)-\right.$ $\left.\left(\beta_{\mathrm{t}} p_{\mathrm{t}}-\beta_{\mathrm{i}} p_{\mathrm{i}}\right)-\left(E_{\mathrm{t}}-E_{\mathrm{i}}\right)\right]$. When the cost of adopting telehealth is above this threshold, patients will abandon it. Therefore, if the threshold value is appropriately raised, the proportion of patients adopting telehealth can be increased. We analyse the threshold composition as follows: first, $m_{\mathrm{i}}-m_{\mathrm{t}}$. Research from the American Telemedicine Association has consistently shown that the quality of telehealth is equal to that provided by in-person visits [46]. However, patients influenced by traditional Chinese medicine usually perceive the effectiveness of telehealth to be lower than that of face-to-face consultations, resulting in a lower threshold. Second, $\delta B_{(\mathrm{d})}$. The closer the patient is to the doctor and the lower the traffic congestion, the smaller is the travel burden of the patient and the lower the threshold. Third, $\beta_{\mathrm{t}} p_{\mathrm{t}}-\beta_{\mathrm{i}} p_{\mathrm{i}}$. Charges for telehealth vary from place to place but are greater than those for face-to-face visits and in most areas not covered by medical insurance [47-49]. In other words, the patient bears more of the cost in the telehealth mode than in the face-to-face mode, resulting in a lower threshold. Finally, $E_{\mathrm{t}}-E_{\mathrm{i}}$. Since telehealth services are still in their infancy, they are coordinated by parttime staff assigned by participating hospitals. The low operating efficiency leads to long waiting times and further lowers the threshold $[49,50]$. It is not difficult to see that, by popularizing telehealth knowledge, promoting telehealth in areas far from doctors and with inconvenient transportation, implementing the same fees and medical insurance benefits as for face-to-face services, and improving operational efficiency, the threshold can be further simplified to $\alpha B_{(\mathrm{d})}$. That is, when the right policies and measures are put in place and no new learning costs are incurred after patient training, $S_{\mathrm{v}}=0$, patients will eventually adopt telehealth if the patient's amortized cost of telehealth is less than the product of the telehealth feasibility and travel burden values.

\section{Conclusions}

In the context of an intensive push to spread telehealth coverage in China, the adoption rate of telehealth remains 
low due to the conflicts between the habits formed by doctors and patients under the influence of the strong traditional Chinese medicine culture and telehealth. The current study analysed the factors influencing doctors' and patients' adoption of telehealth, constructed an asymmetric replication dynamic evolutionary game model, analysed doctors' and patients' visit behaviours, and derived evolutionarily stable strategies in different situations. The results show that doctors' utility from using telehealth is low and the cost threshold of patients using telehealth is also low. This "dual low" nature of telehealth directly leads doctors and patients not to adopt it.

To promote the adoption of telehealth in China, key options include providing adequate training for doctors and patients in telehealth technology, rewarding doctors, and raising patients' cost threshold for adopting telehealth, such that the utility of doctors and patients from adopting telehealth becomes higher than their utility from habitual inperson visits. In addition, doctors adopting telehealth is a prerequisite for patients adopting telehealth. The rewards to doctors should be extended until doctors can obtain the same remuneration from telehealth consultations as from face-to-face visits, and thus no new learning costs are incurred after they are trained in telehealth use. In regards to patients, they will eventually adopt telehealth with the implementation of appropriate policies and measures, such as popularizing telehealth knowledge, promoting telehealth in areas far from doctors and with inconvenient transportation, setting the same fees and medical insurance benefits as those for face-to-face treatment, improving operational efficiency, and ensuring that no additional learning costs are incurred after patients receive telehealth training. Moreover, the patient's amortized cost of telehealth should be less than the product of the telehealth feasibility and travel burden values.

The study is likely to help developing countries solve the problem of conflicts between telehealth and traditional culture and habits in the process of telehealth promotion campaigns by stressing that the failure to adopt telehealth stems from doctors and patients obtaining less utility from telehealth visits than from in-person visits. The study can serve as a reference to help developing countries promote the adoption of telehealth through careful analysis of the factors hampering the promotion and adoption of telehealth.

\section{Data Availability}

The data used to support the findings of this study are available from the corresponding author upon reasonable request.

\section{Conflicts of Interest}

The authors declare that they have no conflicts of interest.

\section{Acknowledgments}

This work was supported by the National Natural Science Foundation of China (Grant nos. 71671091 and 41801119).

\section{References}

[1] E. R. Dorsey and E. J. Topol, "State of telehealth," New England Journal of Medicine, vol. 375, no. 2, pp. 154-161, 2016.

[2] J. Kvedar, M. J. Coye, and W. Everett, "Connected health: a review of technologies and strategies to improve patient care with telemedicine and telehealth," Health Affairs, vol. 33, no. 2, pp. 194-199, 2014.

[3] S. Delaigue, L. Bonnardot, O. Steichen et al., "Seven years of telemedicine in médecins sans frontières demonstrate that offering direct specialist expertise in the frontline brings clinical and educational value," Journal of Global Health, vol. 8, no. 2, Article ID 020414, 2018.

[4] T.-T. Wang, J.-M. Li, C.-R. Zhu et al., "Assessment of utilization and cost-effectiveness of telemedicine program in western regions of China: a 12-year study of 249 hospitals across 112 cities," Telemedicine and E-Health, vol. 22, no. 11, pp. 909-920, 2016.

[5] C. Combi, G. Pozzani, and G. Pozzi, "Telemedicine for developing countries," Applied Clinical Informatics, vol. 07, no. 04 , pp. 1025-1050, 2016.

[6] A. G. Ekeland, A. Bowes, and S. Flottorp, "Effectiveness of telemedicine: a systematic review of reviews," International Journal of Medical Informatics, vol. 79, no. 11, pp. 736-771, 2010.

[7] Policy $\mathrm{OOH}$, "Report to congress: e-health and telemedicine," 2016, https://aspe.hhs.gov/system/files/pdf/206751/TelemedicineEHealthReport.pdf.

[8] WHO, "Global diffusion of eHealth: making universal health coverage achievable: report of the third global survey on eHealth," 2016, https://www.who.int/goe/publications/global_ diffusion/en/.

[9] M. Zhou, L. Zhao, N. Kong, K. S. Campy, S. Qu, and S. Wang, "Factors influencing behavior intentions to telehealth by Chinese elderly: an extended TAM model," International Journal of Medical Informatics, vol. 126, pp. 118-127, 2019.

[10] X. Jinping, "National health conference: put people's health as a priority development strategy and work hard to protect people's health in all aspects," 2016, https://www.who.int/goe/ publications/global_diffusion/en/.

[11] W. Junping, "Making telemedicine fair and efficient," 2019, https://www.who.int/goe/publications/global_diffusion/en/.

[12] C. M. LeRouge, M. Gupta, G. Corpart, and A. Arrieta, "Health system approaches are needed to expand telemedicine use across nine Latin American nations," Health Affairs, vol. 38, no. 2, pp. 212-221, 2019.

[13] Asia GHFoBFf, "Innovation promotes health, and the future of internet healthcare faces major opportunities," 2019, http:// health.people.com.cn/n1/2019/0611/c14739-31129870.html.

[14] H. Li, K. Liu, J. Gu, Y. Zhang, Y. Qiao, and X. Sun, "The development and impact of primary health care in China from 1949 to 2015: a focused review," The International Journal of Health Planning and Management, vol. 32, no. 3, pp. 339-350, 2017.

[15] X. Jinping, "Report delivered at the 19th national congress of the communist party of China," 2017, http://www.xinhuanet. com/english/special/2017-11/03/c_136725942.htm.

[16] Asia GHFoBFf, "China's medical talent team construction should work hard at the grassroots level," 2019, http://www. ghfbfa.cn/newsDetail/?id=258.

[17] M. Wei, "China's communications infrastructure capacity has grown by leaps and bounds," 2018, https://baijiahao.baidu. $\mathrm{com} / \mathrm{s}$ ? id $=1612678610880277436 \& w f r=$ spider $\& f o r=p c$.

[18] China NHaFPCo, "Technical guide for telemedicine information system construction," 2014, http://www.nhc.gov.cn/guihuaxxs/ s10741/201501/e023e2c4e3254f73932f0b0fca99a866.shtml. 
[19] Y. Jie, "Promote telemedicine and establish industry standards and evaluation systems," 2019, http://www.jnlc.com/article/ 20190308236829.shtml.

[20] Commission NH, "Statistical communique on the development of China's health undertakings in 2018," 2019, http://www.nhc.gov. cn/guihuaxxs/s10748/201905/9b8d52727cf346049de8acce25ffcbd0. shtml.

[21] M. J. S. Diño and A. B. de Guzman, "Using partial least squares (PLS) in predicting behavioral intention for telehealth use among Filipino elderly," Educational Gerontology, vol. 41, no. 1, pp. 53-68, 2015.

[22] Y. Xue and H. Liang, "Analysis of telemedicine diffusion: the case of China," IEEE Transactions on Information Technology in Biomedicine, vol. 11, no. 2, pp. 231-233, 2007.

[23] K. I. Adenuga, N. A. Iahad, and S. Miskon, "Towards reinforcing telemedicine adoption amongst clinicians in Nigeria," International Journal of Medical Informatics, vol. 104, pp. 84-96, 2017.

[24] B. Kamsu-Foguem and C. Foguem, "Could telemedicine enhance traditional medicine practices?" European Research in Telemedicine/La Recherche Européenne en Télémédecine, vol. 3, no. 3, pp. 117-123, 2014.

[25] E. Ostrom, J. Burger, C. B. Field et al., "Revisiting the commons: local lessons, global challenges," Science, vol. 284, no. 5412, pp. 278-282, 1999.

[26] E. Fehr and U. Fischbacher, "The nature of human altruism," Nature, vol. 425, no. 6960, pp. 785-791, 2003.

[27] J. Von Neumann and O. Morgenstern, Theory of Games and Economic Behavior, Princeton University Press, Princeton, NJ, USA, 1944.

[28] J. M. Smith, "Evolution and the theory of games," American Scientist, vol. 64, pp. 41-45, 1976.

[29] N. Zhang, X. Zhang, and Y. Yang, "The behavior mechanism of the urban joint distribution alliance under government supervision from the perspective of sustainable development," Sustainability, vol. 11, no. 22, p. 6232, 2019.

[30] S. Dünnebeil, A. Sunyaev, I. Blohm, J. M. Leimeister, and H. Krcmar, "Determinants of physicians' technology acceptance for e-health in ambulatory care," International Journal of Medical Informatics, vol. 81, no. 11, pp. 746-760, 2012.

[31] C.-F. Liu, Y.-C. Tsai, and F.-L. Jang, "Patients' acceptance towards a web-based personal health record system: an empirical study in Taiwan," International Journal of Environmental Research and Public Health, vol. 10, no. 10, pp. 5191-5208, 2013.

[32] C.-H. Tsai, "Integrating social capital theory, social cognitive theory, and the technology acceptance model to explore a behavioral model of telehealth systems," International Journal of Environmental Research and Public Health, vol. 11, no. 5, pp. 4905-4925, 2014.

[33] M. J. Rho, I. y. Choi, and J. Lee, "Predictive factors of telemedicine service acceptance and behavioral intention of physicians," International Journal of Medical Informatics, vol. 83, no. 8, pp. 559-571, 2014.

[34] Y. Wang, Patient Choice of Service Pattern in the Healthcare System, Southwestern University of Finance and Economics, Chengdu, China, 2016.

[35] V. Venkatesh, M. G. Morris, G. B. Davis et al., "User acceptance of information technology: toward a unified view," MIS Quarterly, vol. 27, no. 13, pp. 425-478, 2003.

[36] C.-L. Lee, D. C. Yen, K.-C. Peng, and H.-C. Wu, "The influence of change agents' behavioral intention on the usage of the activity based costing/management system and firm performance: the perspective of unified theory of acceptance and use of technology," Advances in Accounting, vol. 26, no. 2, pp. 314-324, 2010.

[37] B. Rajan, T. Tezcan, and A. Seidmann, "Service systems with heterogeneous customers: investigating the effect of telemedicine on chronic care," Management Science, vol. 65, no. 3, pp. 1236-1267, 2019.

[38] X. Wang, R. Du, S. Ai et al., "The evolution analysis of the community hospitals and patients' behavior selection under the background of telemedicine," Industrial Engineering and Management, vol. 20, pp. 130-137, 2015.

[39] X. Zhan, L. Zhou, and X. Sun, "Promoting telemedical services based on evolutionary game theory," Systems Engineering, vol. 35, pp. 95-102, 2017.

[40] C. Scott Kruse, P. Karem, K. Shifflett, L. Vegi, K. Ravi, and M. Brooks, "Evaluating barriers to adopting telemedicine worldwide: a systematic review," Journal of Telemedicine and Telecare, vol. 24, no. 1, pp. 4-12, 2018.

[41] S. Xie, Economic Game Theory, Fudan University Press, Shanghai, China, 2002.

[42] D. Friedman, "Evolutionary games in economics," Econometrica, vol. 59, no. 3, pp. 637-666, 1991.

[43] C. Ranganathan and S. Balaji, "Key factors affecting the adoption of telemedicine by ambulatory clinics: insights from a statewide survey," Telemedicine and E-Health, vol. 26, no. 2, pp. 218-225, 2020.

[44] J. Moeckli, P. Cram, C. Cunningham, and H. S. Reisinger, "Staff acceptance of a telemedicine intensive care unit program: a qualitative study," Journal of Critical Care, vol. 28, no. 6, pp. 890-901, 2013.

[45] R. Bhatta, K. Aryal, and G. Ellingsen, "Opportunities and challenges of a rural-telemedicine program in Nepal," Journal of Nepal Health Research Council, vol. 13, pp. 149-153, 2015.

[46] Association TAT, "The American telemedicine association reports that telehealth/telemedicine has been growing rapidly because it offers four fundamental benefits," 2020, http:// sctelehealth.org/About/.

[47] G. J. Chen and L. Yuan, "Jiangsu telemedicine service has standards,” 2018, http://js.people.com.cn/n2/2018/1015/ c360306-32158596.html.

[48] F. Yao, C. Yunfen, W. Nina et al., "Yesterday, Jiangsu medical reform implemented on the first day, cheap or expensive to see a doctor," 2015, https://js.qq.com/a/20151101/008541.htm.

[49] Asia GHFoBFf, "Telemedicine now has a problem of "applauding or not"," 2019, http://finance.sina.com.cn/roll/ 2019-06-11/doc-ihvhiqay4769923.shtml.

[50] B. Wang, "How long is the road to popularize "telemedicine", 2018, http://www.sohu.com/a/233240219_161795. 